\title{
AN EXPERT GAMIFICATION SYSTEM FOR VIRTUAL AND CROSS-CULTURAL SOFTWARE TEAMS
}

\author{
Isaac Chow ${ }^{1,2}$ and LiGuo Huang ${ }^{2}$ \\ ${ }^{1} \mathrm{XO}$ Communications, USA \\ ${ }^{2}$ Department of Computer Science, Southern Methodist University, \\ Dallas, TX 75275-0122, USA \\ isaac.chow@xo.com; lghuang@lyle.smu.edu
}

\begin{abstract}
Gamification is the concept of applying game elements in non-game context platforms to motivate people to participate in planned activities to achieve goals.

Gamification has been applied to academic fields including software engineering (SE) in recent years. Many gamification implementations in SE have been ad hoc and lacked standardized guidelines. This paper introduces a new concept of building an expert gamification system (EGS) to provide guidelines for the implementation of gamification for virtual and cross cultural software teams (VCCST). The system will extend the core of a regular expert system to include gamification tools, a supplementary database, and an expert knowledge source. The cross-cultural data for the EGS contains the Hofstede's cultural dimensions (HCD).
\end{abstract}

As more and more VCCST are formed in recent years, many issues have been raised in those teams stemming from miscommunication and cultural conflicts. This paper uses the EGS to help resolve the issues in VCCST.

\section{KEYWORDS}

Gamification, Software Engineering, Expert System, Hofstede's Cultural Dimensions, \& Theory of Flow

\section{INTRODUCTION}

Gamification is the use of elements of game design in non-game contexts [1]. It is the application of game elements used to encourage engagement with a product or service. The concept of gamification has been around for more than a decade. There has been a great deal of commercial and academic interest on the use of gamification in recent years.

Some studies show that gamification can motivate engineers in SE if applied appropriately. However, many gamification implementations for SE are lacking a well-defined framework or guidelines. This paper develops an EGS that will provide guidelines for implementing gamification for VCCST.

An expert system is a computer system that emulates the decision-making ability of a human expert. Expert systems are designed to solve complex problems by reasoning about knowledge

David C. Wyld et al. (Eds) : CSITA, ISPR, ARIN, DMAP, CCSIT, AISC, SIPP, PDCTA, SOEN - 2017 pp. 147-157, 2017. (C) CS \& IT-CSCP 2017

DOI : $10.5121 /$ csit.2017.70115 
[2]. This paper extends the core of an expert system to include gamification tools, a supplementary database with the cross-cultural data, and the expert knowledge source from theory of flow to build the EGS. The core of an expert system consists of the inference engine and the knowledge base. Figure 1 is a high-level view of the EGS.

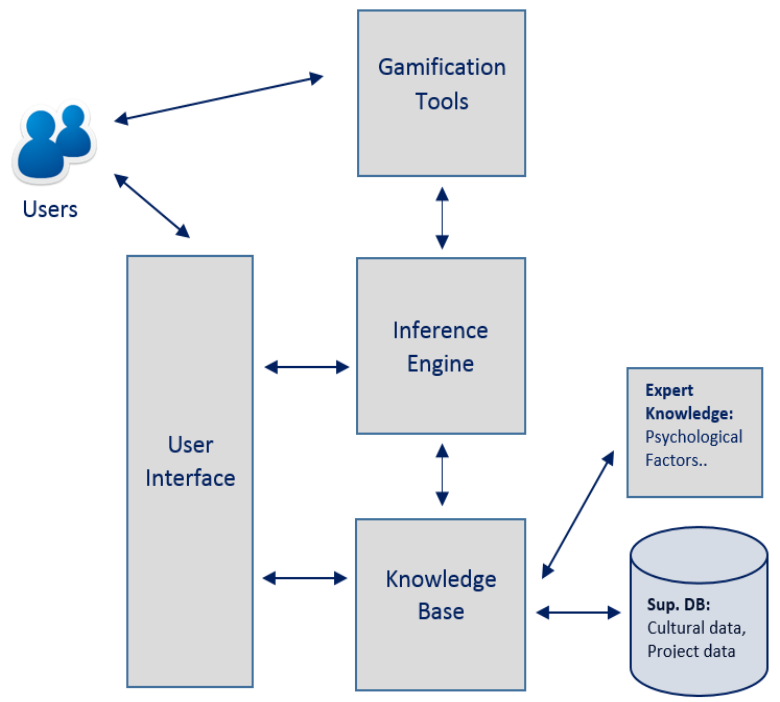

Figure 1. High Level View of the EGS

The EGS consists of the following modules:

1. Gamification Tools

2. User Interface

3. Inference Engine

4. Knowledge Base

5. Supplementary Database

6. Expert Knowledge Source

\section{RELATED WORK}

Gamification has been extensively researched by different types of researchers in recent years. However, there are very few studies in building an EGS as a tool for implementing gamification. Some gamification studies are intended for learning and discussed in the context of education. Other work tried to verify whether or not gamification works [3]. Some analysis has been done to investigate the gamification mechanisms and see how gamification influences behaviours [4]. Some research has indicated that gamification provides positive effects in motivation. Some studies are descriptive in nature and they have no actual listed experiment results of gamification reported [5], [6], [7], [8]. Several studies explore conceptual frameworks for classroom learning only [9].

For VCCST, some studies describe their conflicts and issues in detail but offer little help or guidance for them [10], [11]. Other research identifies the specific attributes relevant to crosscultural concerns but does not show how to resolve the issues [12]. Some studies describe how to do cross-cultural surveys [13]. There are very few studies offering solutions for using an EGS. 


\section{Virtual AND Cross Cultrual Software Team}

Globalization of markets has been a major theme in recent years and more software teams are working in cross cultural environments from outsourcing or insourcing work strategies [10]. It is common for a large software project to have teams in more than one location and in different countries. Many VCCST are formed across the globe with engineers from different parts of the world [14]

It has been reported that the software projects with VCCST have quite a few issues and challenges.Most of the problems are about conflicts in culture and its adverse impact to the dynamics of the team [10], [15]. In a few instances, the software engineers view their co-workers from different cultures as rivals rather than teammates. This paper designs an EGS to promote team work and collaboration for VCCST to smoothen their conflicts and problems.

\section{EXPERT GAMIFCATION SYSTEM}

The EGS extends the core of an expert system and consists of six components as shown in Figure 1. The core elements of an expert system are the inference engine and the knowledge base. The inference engine is an automated reasoning system that evaluates the current state of the knowledge base, applies relevant rules, and then asserts new knowledge into the knowledge base. The knowledge base represents facts and rules. Knowledge in an expert system may originate from many sources such as reports, databases, case studies, empirical data, and domain experts [2].

Other components in the EGS are the gamification tools, a user interface, a supplementary database, and an expert knowledge source. The gamification tools include the game mechanics and the platform. For example, Microsoft SharePoint can be used as a gamification tool. These tools will contain the software team profile and personal profiles. The profiles will store and show the team scores and individual scores respectively. The user interface facilitates the communications between the project admins, users, and the EGS.

The supplementary database contains the cultural data and project data. The expert knowledge source contains the theory of flow information. The following two sections describe the cultural data and the theory of flow for this EGS.

\subsection{Hofstede's cultural dimensions}

Hofstede's cultural dimensions (HCD) theory is a framework for cross-cultural communication. It describes the effects of a society's culture on the values of its members, and how these values relate to behaviour using a structure derived from factor analysis [16]. According to Hofstede the most important differences between cultures can be captured by finding to what extent members of these cultures differ with regard to six values [17]:

1. Power distance index (PDI) - The power distance index is defined as the extent to which less powerful people in an organization will accept and expect power to be distributed.

2. Individualism/Collectivism (IDV) - Differences between the degrees within the Individualism vs. Collectivism index.

3. Masculinity/Femininity (MAS) - Differences between the degrees within the Masculinity vs. Femininity index. 
4. Uncertainty avoidance index (UAI) - Differences between the degrees within the Uncertainty Avoidance Index.

5. Long-term/Short-term Orientation (LTO) - Differences between the degrees within the Long-Term vs. Short-Term Orientation index.

6. Indulgence vs. Restraint (IND) - Differences between the degrees within the Indulgent vs. Restraint index.

Table 1 shows the Hofstede's values for these cultural dimensions for Australia, China, India, Japan, Netherlands, UK, and USA [17]. The EGS further categorizes the countries into regions such as Australia (AUS), Asia, Europe (EU), and North America (NAM) for further region processing as shown in the second columns of Table 1.

Table 1. Hofstede's Cultural Dimensions

\begin{tabular}{|l|l|l|l|l|l|l|l|}
\hline Country & Region & PDI & INV & MAS & UAI & LTO & IND \\
\hline Australia & AUS & 36 & 90 & 61 & 51 & 31 & 71 \\
\hline China & Asia & 80 & 20 & 66 & 30 & 87 & 24 \\
\hline India & Asia & 77 & 48 & 56 & 40 & 51 & 26 \\
\hline Japan & Asia & 50 & 42 & 90 & 88 & 75 & 42 \\
\hline Netherlands & EU & 38 & 80 & 14 & 53 & 67 & 68 \\
\hline UK & EU & 30 & 89 & 62 & 30 & 20 & 69 \\
\hline USA & NAM & 40 & 91 & 62 & 46 & 26 & 68 \\
\hline
\end{tabular}

Among the HCD, three dimensions have more effect on gamification. They are Individualism, Masculinity, and Uncertainty:

\section{Individualism (INV)}

This is the index to indicate how people think with the mentality of "I" or "We." Some countries are very "individual" focused. They focus more on themselves and careers versus their group. The game design should take this into consideration. If the culture is more individual focused, game mechanics such as leaderboards should be used.

\section{Masculinity (MAS)}

Masculinity is defined as "a preference in society for achievement, heroism, assertiveness and material rewards for success." Its counterpart represents "a preference for cooperation, modesty, caring for the weak and quality of life." [17] This dimension gives important information in setting up the right game mechanics for certain cultures. For instance, many Scandinavian countries have a very low score in MAS. In those cultures, there is an important concept known as Janteloven [8]. Janteloven is essentially a set of rules for encouraging social equality, social stability, and uniformity in which one should never try to stick out from the crowd. The Netherlands is an example with a score of 14 in MAS. The EGS should take this into consideration by not focusing the games on competitiveness and leaderboards. Rather, it should promote notions of equality and creativity for them [18], [19].

\section{Uncertainty Avoidance (UAI)}

The uncertainty avoidance index is defined as "a society's tolerance for ambiguity" in which people embrace something unexpected, or go away from the status quo. Societies that score a high degree in this index tend to prefer strict codes of behaviour, guidelines, laws, and absolute 
Truth. A lower degree in this index shows more acceptance of differing thoughts. Society tends to impose fewer regulations, ambiguity is more accustomed to [17]. The game design should take this into consideration.

\subsection{Psychological factors and flow detection}

Gamification uses the psychology of engagement to motivate people. Psychology can be applied to the non-technical aspects of SE like personality, teamwork, leadership, decision-making, culture, motivation and human tendencies [15].

Abraham H. Maslow was a renowned psychologist in the field of motivation. He published the famous Hierarchy of Needs in 1943 [18]. It is a pyramid depicting the five levels of needs, namely

1. Biological and Physiological: air, food, shelter, water, sex, \& rest

2. Safety: health, protection, stability, freedom from fear \& security

3. Love and Belonging: love, intimacy, friendship, family, \& affiliating

4. Esteem: self-esteem, independence, status, confidence, achievement \& respect

5. Self-actualization: realizing personal potential, self-fulfilment \& peak experiences

Self-actualization is the peak level in which a person's full potential is realized. Maslow describes this level as the desire to accomplish everything that one can: to become the most that one can be [18].

\subsubsection{Theory of flow and group flow}

Self-actualized people are those who come to find meanings to life that are important to them and that fulfil them. Many of them experience a common phenomenon called flow, proposed by Csikszentmihalyi [20].When people are in a state of flow, they will feel focused and concentrated. They may feel a sense of ecstasy, great inner clarity, a sense of serenity, timelessness, and intrinsic motivation. The flow is the balance between the skill level and the challenge of a task. When a task is too difficult, it causes people to worry. When a task is too simple, people will be bored. When a task is balanced between skill and difficulty, the individual will reach a state of heightened focus and immersion [20]. Some research further extends the flow theory for groups. Keith Sawyer developed specific triggers needed for creating group flow [21]. Figure 2 shows the theory of flow. The EGS should try to keep the team in the flow and should be able to detect the flow zone.

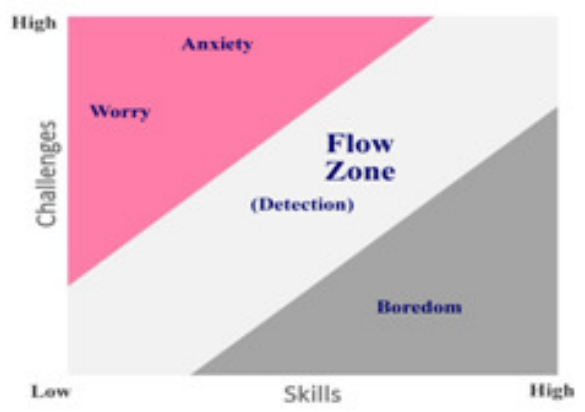

Figure 2. Flow Theory 


\subsubsection{Flow Zone Detection}

The EGS should find ways to measure and detect the flow zone. There are some methods in measuring flow with questionnaires: Nakamura \& Csikszentmihalyi enlist the various ways of measuring flow for Concept of Flow; Intrinsic Motivation Inventory with 10 Questionnaires; John Marshall Reeve: Agentic Engagement Scale Questionnaires; Davin Pavlas Play Experience Questionnaire [21]. Other than using the questionnaires, the EGS can use the game statistics to detect the flow zone. Figure 3 shows a simple game flow in which the 'flow' loop is indicated with bigger arrows.

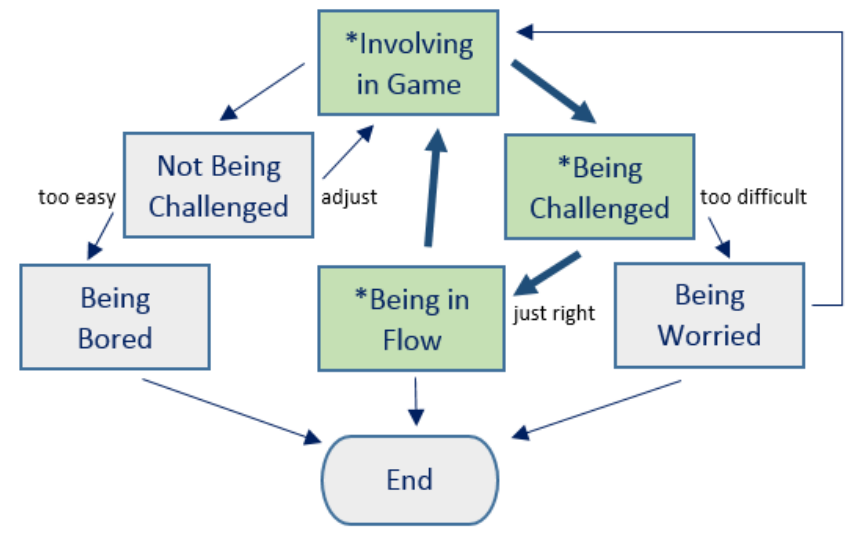

Figure 3. A Simple Game Flow

The EGS should monitor the game and collect the game statistics. The EGS should set up rules to determine if a team is in a flow state. Table 5 shows a sample of the game statistics. The average percentage of hits in that table can be a strong indicator for detecting a flow state. For example, if that percentage is at least at a certain value, then the EGS should assume that the team is in a flow state or zone. It may be a trial and error process to finalize the right value for that percentage. The percentage can be cross checked with the questionnaire method to find the optimal value.

\subsection{Determining Game Mechanics and Activities}

The EGS should extract the cultural information and use that information to configure the game mechanics in the system. Table 2 contains the game mechanics, the associated emotions, and their values for INV, MAS and UAI. The values represent the recommendations from the EGS for the game mechanics. For example, the value of 'all' in INV means that game mechanic is good for all values of INV. The value of 'high' in INV means the game mechanic is good if INV is high. The same rule applies to the value of 'low'. If all three HCDs have the same 'high' value for the same game mechanics, it reinforces the recommendations. If the HCDs have different recommendations, INV will have the highest preference and MAS will have the second highest preference.

Table 2. Game mechanics and cultural factors

\begin{tabular}{|l|l|l|l|l|}
\hline Game mechanics & Emotions & INV & MAS & UAI \\
\hline Points & Reward & all & all & all \\
\hline Badges & Achievement & all & high & all \\
\hline Leaderboards & Competition & high & high & high \\
\hline Levels & Status & high & high & high \\
\hline Progress bars & Achievement & all & all & high \\
\hline Storyline & Engagement & all & all & all \\
\hline Feedback & Engagement & all & all & high \\
\hline
\end{tabular}




\begin{tabular}{|l|l|l|l|l|}
\hline $\begin{array}{l}\text { Challenge/between } \\
\text { users }\end{array}$ & Competition & all & high & all \\
\hline Virtual gifts & Altruism / Self-expression & all & low & low \\
\hline $\begin{array}{l}\text { System for } \\
\text { sharing/exchanging }\end{array}$ & $\begin{array}{l}\text { Collaboration/ } \\
\text { Community }\end{array}$ & low & low & all \\
\hline
\end{tabular}

Table 3 shows a list of activities with their corresponding values for INV, MAS, or UAI. The rules for recommendation are similar to the ones in Table 2. For example, the value of 'low' in INV for 'helping others' means that activity is recommended when it has a low INV value. The same rule applies to other activities and HCDs. Also, the HCD values in the table can be changed when more knowledge or experience is gathered.

Table 3. Activity table with cultural factors

\begin{tabular}{|l|l|l|l|}
\hline Activities & INV & MAS & UAI \\
\hline Inviting peers to design review & all & all & all \\
\hline Participating in a design review & all & all & high \\
\hline Inviting peers to a code review & all & all & all \\
\hline Participating in a code review & all & all & all \\
\hline Inviting peers to a test plan review & all & all & all \\
\hline Participating in a test plan review & all & all & all \\
\hline Helping others & low & low & all \\
\hline Giving people credits & low & low & all \\
\hline Being a team player & low & low & all \\
\hline Resolving issues & all & low & high \\
\hline Collaboration activities & low & low & all \\
\hline Innovative idea & all & all & all \\
\hline Voting for an idea & low & low & all \\
\hline Beating the deadline & high & all & high \\
\hline Documenting code & all & all & high \\
\hline Best practice & all & all & high \\
\hline Writing unit tests & all & all & high \\
\hline
\end{tabular}

\subsection{Sample Data}

A simple prototype is developing at this time. The preliminary findings are encouraging. Table 4 contains some sample inputs to the EGS for demo purpose. These inputs drive the EPS based rules mentioned in 4.3 and the 4.2.2 example.

Table 4. Inputs to the system

\begin{tabular}{|l|l|}
\hline Attributes & Examples \\
\hline Project Name & VCCST Enhancement \\
\hline Project Description & A demo to show how the EGS works \\
\hline Domain of interest & Software engineering \\
\hline Team Info & Cross-Cultural \\
\hline Team Location & Virtual \\
\hline Countries & USA, India, China \\
\hline \# of team members & $20,12,18$ \\
\hline \# of analyst, developers, testers & $4,12,4$ \\
\hline SE Methodology & Agile \\
\hline
\end{tabular}


The ESG will recommend a list of game mechanics and activities for the software project. Each individual group is customized. The EGS collects statistics from the project for analysis. One of the important tasks is to detect the flow zone for the software project team.

Table 5 shows a sample of the game statistics. The EGS uses the values from INV, MAS, UAI, and the team cultural data to determine the initial 'target \%' for the flow zone. As more project data are stored and accumulated, the EGS can use the past data as reference and knowledge for further 'target \%' refinement and fine tuning. If the HCD recommend the activities, the 'target $\%$ ' for those activities will be higher than others.

Table 5. Sample game statistics

\begin{tabular}{|c|c|c|c|c|}
\hline Point for activities & $\begin{array}{l}\text { \# of } \\
\text { players }\end{array}$ & $\begin{array}{l}\text { \# of } \\
\text { hits }\end{array}$ & Hit \% & $\begin{array}{l}\text { Target } \\
\text { hit } \%\end{array}$ \\
\hline inviting peers to design review & 16 & 15 & 93.75 & 80.00 \\
\hline participating in a design review & 16 & 9 & 56.25 & 80.00 \\
\hline inviting peers to a code review & 12 & 8 & 66.67 & 80.00 \\
\hline participating in a code review & 12 & 6 & 50.00 & 80.00 \\
\hline inviting peers to a test plan review & 16 & 7 & 43.75 & 80.00 \\
\hline participating in a test plan review & 16 & 4 & 25.00 & 80.00 \\
\hline helping others & 20 & 6 & 30.00 & 70.00 \\
\hline giving people credits & 20 & 15 & 75.00 & 75.00 \\
\hline being a team player & 20 & 9 & 45.00 & 75.00 \\
\hline resolving issues & 20 & 5 & 25.00 & 70.00 \\
\hline collaboration activities & 20 & 12 & 60.00 & 70.00 \\
\hline innovative idea & 20 & 3 & 15.00 & 60.00 \\
\hline voting for an idea & 20 & 4 & 20.00 & 70.00 \\
\hline beating the deadline & 20 & 4 & 20.00 & 80.00 \\
\hline documenting code & 12 & 8 & 66.67 & 80.00 \\
\hline best practice & 16 & 5 & 31.25 & 75.00 \\
\hline writing unit tests & 12 & 11 & 91.67 & 80.00 \\
\hline Average \%: & & & 47.94 & 75.59 \\
\hline
\end{tabular}

\begin{tabular}{|l|r|}
\hline A: Number of analysts & 4 \\
\hline B: Number of developers & 12 \\
\hline C: Number of testers & 4 \\
\hline D: Total players & 20 \\
\hline
\end{tabular}

In Table 5, the 'Hit \%' is calculated by '\# of hits' / '\# of players'. In this sample data, there are 4 analysts, 12 developers, and 4 testers - a total of 20 players. The system assigns players to the activities according to their roles. The system collects the games statistics including the number of hits from each activity. The system compares the average 'Hit \%' and the average 'Target hit $\%$ ' to determine the status of the flow zone. In Table 5, 47.94\% is the average 'Hit \%' and $75.59 \%$ for the average 'Target hit \%'. Since the average 'Hit \%' is lower than the average 'Target hit \%', the EGS will infer that the project team is not in a flow zone. The EGS will send alerts to the system admins and project managers. They can choose to adjust the game mechanics/activities and or make comments about the project. The project data will be stored and served as reference and knowledge data for future projects. A SharePoint community site will be used as a platform for collaboration, community, and data collection. 


\section{CONCLUSIONS}

This paper introduces a new concept for building an EGS for the implementation of gamification for VCCST. The goal of this paper is to open up another way of thinking in designing gamification for SE using cross cultural data, the flow theory, and the past project data for the VCCST. Designing and building the EGS will help to create a user-friendly system tool for gamification implementation. The concept can be further extended to other domains for resolving different problems using gamification.

For future work in this EGS, one area to investigate is how to better detect the state of flow and group flow using the project data or other means. A more formal algorithm should be developed for that. Also, incorporating more cultural data to develop a robust knowledge extraction methodology for the system could be beneficial. As for the VCCST, more case studies should be done using gamification with qualitative and quantitative approaches. Supporting and fostering the gamification research to improve the software development process in the cross-cultural environment could yield impactful results for VCCST's.

\section{REFERENCES}

[1] Deterding, S., Sicart, M., Nacke, L., O'Hara, K. \& Dixon, D. (2011). Gamification: Using game design elements in non-gaming contexts. In Proceedings of CHI 2011. New York: ACM Press, 24252428.

[2] Wikipedia, "Expert system”, (2016), https://en.wikipedia.org/wiki/Expert_system

[3] J Hamari, J Koivisto, H Sarsa, "Does gamification work? -a literature review of empirical studies on gamification" System Sciences (HICSS), 2014, pp. 3024-3034.

[4] Bunchball (2010), "Gamification 101: An introduction to the Use of Game Dynamics to Influence Behavior"

[5] Philipp Herzig, Michael Ameling, Alexander Schill, "A Generic Platform for Enterprise Gamification", 2012 Joint Working Conference on Software Architecture \& 6th European Conference on Software Architecture, 978-0-7695-4827-2/12 @ 2012 IEEE, pp. 217-223.

[6] Foong Li Law, Zarinah Mohd Kasirun, Chun Kiat Gan, "Gamification towards Sustainable Mobile Application", 2011 5th Malaysian Conference in Software Engineering (MySEC), pp. 348-353.

[7] Bogdan Vasilescu, "Human Aspects, Gamification, and Social Media in Collaborative Software Engineering", ICSE '14, May 31 - June 7, 2014, Hyderabad, India, Copyright 14 ACM 978-1-4503$2768-8 / 14 / 05$

[8] Marius Kalinauskas, "GAMIFICATION IN FOSTERING CREATIVITY", ISSN 2029-7564 SOCIAL TECHNOLOGIES 2014, 4(1), pp. 62-75.

[9] Jorge Simões a, Rebeca Díaz Redondo b, Ana Fernández Vilas, "Computers in Human Behavior", www.elsevier.com/locate/comphumbeh, 2012 Elsevier Ltd

[10] Michael Barrett, "Knowledge Sharing in Cross-Cultural Software Teams" Working Paper Series, 18/2007, Judge Business School, University of Cambridge

[11] Hannu Jaakkola, Anneli Heimbürger, "Cross-Cultural Software Engineering", Informatologia 42, 2009., pp. 256-264. 
[12] A Sutharshan. S P Maj "Enhancing Agile Methods for Multi-cultural Software Project Teams" Modern Applied Science Vol. 5, No. 1; February 2011, ISSN 1913-1844 E-ISSN

[13] "Guidelines for Best Practice in Cross-Cultural Surveys" THIRD EDITION, Copyright @ 2011 by the Survey Research Center, Institute for Social Research, University of Michigan, ISBN 978-0-98284181-5, Library of Congress Control Number: 2010937486 2011, pp.163-194.

[14] Christof Ebert, "Collaboration Tools for Global Software Engineering", IEEE SOFTWARE by the IEEE Computer Society, March/April 2010

[15] Marczak, Treude, Filho, Steffens, Singer, Redmiles, "Studying Gamification as a Collaboration Motivator for Virtual Software Teams: Social Issues, Cultural Issues, and Research Methods" CSCW '15 Companion, Mar. 14-18, 2015, Vancouver, BC, Canada. ACM

[16] Wikipedia, "Hofstede's cultural dimensions theory", (2016) https://en.wikipedia.org/wiki/Hofstede\%27s_cultural_dimensions_theory

[17] Geert Hofstede, "Cultural Dimensions", (2010), https://geert-hofstede.com/countries.html

[18] Erica Sahlgren, Kendra Knorst, "Culture's impact on gamification", 2016 ,Linköping University I Department of Management and Engineering, ISRN-number: LIU-IEI-FIL-G--16/01497—SE

[19] Rilla Khaled, "It's Not Just Whether You Win or Lose: Thoughts on Gamification and Culture", CHI 2011, May 7-12, 2011, Vancouver, BC, Canada. ACM 978-1-4503-0268-5/11/05.

[20] Csikszentmihalyi, M, "Flow and the Psychology of Discovery and Invention". Harper, Perennial, New York (1997)

[21] Enterprise Gamification Wiki, "Flow theory", (2015), http://www.enterprise-gamification.com/mediawiki/index.php?title=Flow-theory

\section{AUTHORS}

\section{Isaac Chow}

https://www.linkedin.com/in/isaac-chow-1aa398b

Isaac got his B.S. and M.S. in CS at the University of North Texas in mid 1980's.

Isaac went to teach in Southwestern Oklahoma State University as a computer science

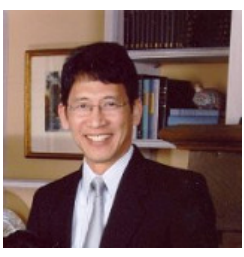
instructor from 1985 to 1989 . Isaac worked for Texas Instruments as a programmer analyst for three years working on mainframe MVS system and received quality recognition and excellence award. He worked for AT\&T for 10 years as a Senior Technical Staff Member and Computer Sciences Corporation for 3 years as a Computer Scientist. He worked on Internet based multi-platform systems using MVS, Unix, C/C++, Java, Sybase, and received the Circle of Excellence award.

Isaac currently works for XO Communications on developing intranet applications using SharePoint, ASP .net, and c\#. Isaac has worked on the Microsoft SharePoint and used it as a collaboration tool for many years. Isaac is a doctoral student in software engineering at SMU. He plans to contribute to the academic community through research and with his intensive software experience. 


\section{Dr. LiGuo Huang}

http://lyle.smu.edu/ lghuang/

Dr. LiGuo Huang Associate Professor, Department of Computer Science and Engineering at the Southern Methodist University (SMU), Dallas, TX, USA. She received both her Ph.D. and M.S. from the Computer Science Department and Center for Systems and Software Engineering (CSSE) at the University of Southern California (USC). Her current research focuses on mining and learning from systems and software engineering repositories, software quality assurance and information dependability, process modelling, simulation and improvement, stakeholder/value-based software engineering, and software metrics. Her research has been supported by NSF, US Department of Defense (DoD) and NSA. She had been intensively involved in initiating the research on stakeholder/value-based integration of systems and software engineering and published related papers in IEEE Computer and IEEE Software. 